\title{
STRONGLY ORE INVARIANT RINGS
}

\author{
AHMED A. M. KAMAL
}

\section{Introduction}

The study of invariant rings started with the work of Coleman and Enochs, who defined a ring $A$ to be invariant if whenever $A[X]$ is isomorphic to $B[X]$ for some ring $B$, then $A$ and $B$ were necessarily isomorphic [3]. Other concepts were also introduced in various works, strong invariance, $n$-invariance and $n$-strong invariance (e.g. [2], [3], [4] and [8]). The class of invariant rings contains a number of important classes of rings, for example local domains, left (right) perfect rings and Von Neumann regular rings are invariant.

Ore invariant rings and strongly Ore invariant rings were defined and studied by Armendariz, Koo and Park [1]. In the present work we continue this study, we call a ring A strongly Ore invariant if whenever the Ore extension $A[X, \sigma, D]$ of $A$ is isomorphic to the Ore extension $B[Y, \rho, \delta]$ of some ring $B$, under an isomorphism $\Psi$, then $\Psi(A)=B$. We attempt in this paper to obtain the conditions under which a ring $A$ to be strongly Ore invariant.

Throughout this paper all rings are associative with unit and the following notation will be preserved. If $A$ is a ring, $\sigma$ will denote an automorphism of $A$ and $D$ a $\sigma$-derivation on $A$ (that is an additive map from $A$ to itself such that $D(a b)=D(a) b+\sigma(a) D(b)$ for all $a, b \in A$ ). The Ore extension $A[X, \sigma, D]$ is the ring of polynomials in $X$ over $A$ with the usual addition and with multiplication subject to the rule $X a=\sigma(a) X+D(a)$ for each $a \in A$. Finally the multiplicative group of units in $A$ is denoted by $A^{*}$, the set of all central idempotents in a ring $A$ is denoted by $B(A)$ and $(B(A))^{\sigma}$ is the set of all $\sigma$-invariant elements of $B(A)$.

\section{Preliminaries}

In this section we collect some well-known facts-concerning $\sigma$-reduced rings and Ore extensions. We also provide a few auxiliary results that are needed throughout this

Received December 10, 1993. 
paper.

A ring $A$ is said to be reduced if $A$ contains no nonzero nilpotent elements and it is $\sigma$-reduced if $X \sigma(X)=0$ implies that $X=0$, for each $X \in A$. Let us remark that if $A$ is a domain (not necessarily commutative), then $A$ is $\sigma$-reduced for each $\sigma \in A u t(A)$. Moreover if $A$ is a reduced ring, then the set of all $\sigma \in A u t(A)$ such that $A$ is $\sigma$-reduced is a normal subgroup of $\operatorname{Aut}(A)$.

(2.1) We remark that $\sigma$-reduced rings are reduced and that their idempotents are $\sigma$-invariant, in the case of regular rings these 2-conditions are sufficient for the ring to be $\sigma$-reduced [7, VI.4.2].

As an immediate consequence of (2.1) we have the following result.

(2.2) Every ideal of a regular $\sigma$-reduced ring $A$ with a $\sigma$-derivation $D$, is a $\sigma$-ideal and $D$-ideal. Furthermore the following statements about an ideal $I$ of $A$ are equivalent

(i) I is a prime ideal.

(ii) I is a $\sigma$-prime ideal.

(iii) I is a D-prime ideal.

Proof. It follows directly from the facts that every ideal of $A$ is an idempotent and every element $a \in I$ can be written in the form a $e$, where $e$ is a central idempotent in A.

It is well-known that if, $A$ is a reduced ring, then $A[X, D]$ is a reduced ring and hence directly finite. This result is special to differential operator rings. The following example illustrates this.

(2.3) Let $K$ be a field. Then $A=K \times K$ is a commutative regular self-injective ring. Define $\sigma: A \rightarrow A$ such that $(a, b) \rightarrow(b, a)$ and let $P(X)=(0,1) X \in A[X, \sigma]$, then $P^{2}=0$.

(2.4) If $A$ is a $\sigma$-reduced ring, then $A[X, \sigma, D]$ is directly finite.

Proof. Since $A$ is $\sigma$-reduced it is reduced, so we can show that $A$ is $\sigma^{n}$-reduced for each positive integer $n$. Therefore $A[X, \sigma, D]$ is a reduced ring and hence directly finite.

(2.5) If $A$ is a semiprime ring, then we have $B(A[X, \sigma, D])=(B(A))^{\sigma}$.

Proof. This is [9, Theorem 3.15].

(2.6) If $A$ is a division ring, then $A[X, \sigma, D]$ is a principal ideal domain.

Proof. This is $[11,1.2 .9$ (ii)]. 


\section{Unit elements in Ore extension rings}

The unit elements of a skew polynomial ring $A[X, \sigma]$ and a differential operator ring $A[X, D]$ over a commutative ring $A$ are investigated in [12] and [5] respectively. In this section we study the unit elements of an Ore extension $A[X, \sigma, D]$, in the case where $A$ is noncommutative ring. We begin by the following lemma.

Lemma 3.1. Let $A$ be a domain (not necessarily commutative). Then the set of all units in $A[X, \sigma, D]$ is the set of all units in $A$.

Proof. Let $f(X)=\sum_{i=0}^{n} a_{i} X^{i}$ and let $g(X)=\sum_{j=0}^{m} b_{j} X^{j}$ be its inverse. Then $a_{n} \sigma^{n}\left(b_{m}\right)=0$, so $a_{n}=0$ or $b_{m}=0$, which implies that $a_{n} X^{n} b_{m} X^{m}=0$. The coefficient of $X^{n+m-1}$ in $f g$ is $a_{n} \sigma^{n}\left(b_{m-1}\right)+a_{n-1} \sigma^{n-1}\left(b_{m}\right)=0$. So we have $b_{m} a_{n-1} \sigma^{n-1}\left(b_{m}\right)=$ 0 . Therefore $b_{m}=0$ or $a_{n-1}=0$ and hence $a_{n} \sigma^{n}\left(b_{m-1}\right)=0$ which again implies $a_{n} X^{n} b_{m-1} X^{m-1}=0$. Continuing this way we find $a_{n} X^{n} b_{k} X^{k}=0$ for all $k, 0 \leq k \leq m$. Thus if we set $f_{i}(X)=\sum_{i=0}^{n-1} a_{i} X^{i}$, then $f_{i}(X) g(X)=1$. Since $A$ is a domain, (2.4) implies that $A[X, \sigma, D]$ is directly finite. Therefore $f_{1}(X)=(g(X))^{-1}=f(X)$. So $f(X)$ must be in $A$ and similarly $g(X)$ is in $A$.

It is well-known that, for each $a \in A, X^{n} a=\sum_{i=0}^{n}\left(\begin{array}{c}n \\ i\end{array}\right) D^{i}(a) X^{n-2}$ in the differential operator ring $A[X, D]$. To obtain a similar formula for the Ore extension $\operatorname{ring} A[X, \sigma, D]$, we need the condition that $\sigma$ and $D$ commute. This condition is used in different works concerning Ore extension rings, for example, see [10]. Also with applying that condition, the skew derivation $(\sigma, D)$ becomes a special case of $q$-skew derivations (quantized derivations) see [6].

Lemma 3.2. Let $A$ be a ring such that $\sigma$ and $D$ commute. Then for each $a \in A$ we have $X^{n} a=\sum_{i=0}^{n}\left(\begin{array}{c}n \\ i\end{array}\right) \sigma^{n-i} D^{i}(a) X^{n-i}$ in the Ore extension $A[X, \sigma, D]$.

Proof. Routine.

Notice that lemma 3.2 is not true if $\sigma$ and $D$ do not commute as the following example shows.

Example 3.3. Let $A$ be the ring $Z_{3} \oplus Z_{3}$ with the usual addition and multiplication defined by $(a, b)(c, d)=(a c, a d+b c)$. Let $\sigma(a, b)=(a, 2 b)$ and $D(a, b)=(b, b)$ for each $(a, b) \in A$. Then $\sigma \in A u t(A)$ and $D$ is a $\sigma$-derivation on $A$. It is clear that $\sigma$ and $D$ do not commute and one can easily show that $X^{2}(1,2) \neq \sum_{i=0}^{2} \sigma^{2-i} D^{2}(1,2) X^{2-\imath}$ as desired.

Proposition 3.4. Let $A$ be a $\sigma$-reduced ring such that $\sigma$ and $D$ commute. Then the set of all units in $A[X, \sigma, D]$ is the set of all units in $A$.

Proof. Since $A$ is a reduced ring, then $a b=0$ implies that $b a=0$ for each $a, b \in A$. If $a b=0$, then $\sigma(a) D(b)=-D(a) b$ and $b \sigma(a) \sigma(b \sigma(a))=0$. But $A$ is $\sigma$ reduced, so $b \sigma(a)=0$ and hence $\sigma(a) D(b)=0 . \sigma(a) \sigma D(b) \sigma(\sigma(a) \sigma D(b))=0$ implies that 
$a D(b)=0$ and so $a \sigma D(b)=0$. Continuing this process we obtain, $a b=0$ implies that $a \sigma^{n} D^{m}(b)=0$ and $\sigma^{n} D^{m}(a) b=0$ for each $n, m=0,1,2, \ldots$. Let $f(X)=\sum_{i=0}^{n} a_{i} X^{i}$ and let $g(X)=\sum_{j=0}^{m} b_{j} X^{j}$ be its inverse. Then $a_{n} \sigma^{n}\left(b_{m}\right)=0$, as $a^{\prime} b_{m}=0$, where $a_{n}=\sigma^{n}\left(a^{\prime}\right)$. But $a^{\prime} b_{m}=0$ implies that $\sigma^{k}\left(a^{\prime}\right) b_{m}=0$ and $b_{m} \sigma^{k}\left(a^{\prime}\right)=0$ for each $k=0,1,2, \ldots$ In particular $a_{n} b_{m}=0$, therefore by using lemma 3.2 ,

$$
a_{n} X^{n} b_{m} X^{m}=a_{n}\left(X^{n} b_{m}\right) X^{m}=\sum_{k=0}^{n}\left(\begin{array}{l}
n \\
k
\end{array}\right) a_{n} \sigma^{n-k} D^{k}\left(b_{m}\right) X^{n+m-k}=0 .
$$

Continuing this way we have $a_{n} X^{n} b_{k} X^{k}=0$ for all $k, 0 \leq k \leq m$ and so $f(X)$ and $g(X)$ must be in $A$ as in the proof of lemma 3.1 .

\section{Strongly Ore invariant rings}

With the aid of the preceding sections we shall now study strong Ore invariance. We begin by the following result which is useful in the sequel.

Proposition 4.1. Let $A$ be a division ring. If $A[X, \sigma, D] \cong B[Y, \rho, \delta]$ via $\Psi$, then $\Psi(A)=B$.

Proof. Since $\Psi\left((A[X, \sigma, D])^{*}\right)=(B[Y, \rho, \delta])^{*}$, then by using (2.6) and lemma 3.1, it follows that $\Psi\left(A^{*}\right)=B^{*}$. Thus $\Psi(A) \subseteq B$.

If $\Psi(X)=b_{0}+b_{1} Y+\cdots+b_{k} Y^{k}$ for some $b_{i}^{\prime} \in B$ with $b_{k} \neq 0$, then $k \geq 1$. Now suppose $\beta_{0}+\beta_{1} \Psi(X)+\cdots+\beta_{n}(\Psi(X))^{n}=0$ for some $\beta_{i} \in B$. Then the highest term in the left-hand expression is $\beta_{n} b_{k} \rho^{k}\left(b_{k}\right) \rho^{2 k}\left(b_{k}\right) \cdots \rho^{(n-1) k}\left(b_{k}\right)$ and so $\beta_{n}=0$. Continuing this way we find $\beta_{0}=\beta_{1}=\cdots=\beta_{n}=0$. Therefore $\left\{1, \Psi(X), \ldots,(\Psi(X))^{n}, \ldots\right\}$ is a left linearly independent set over $B$ and so it easy to show that $\Psi(A)=B$.

Lemma 4.2. Let $A$ be a $\sigma$-reduced ring. Then the set of all idempotents in $A[X, \sigma, D]$ is equal to the set of all idempotents in $A$.

Proof. Follows from (2:1), (2.5) and the fact that every idempotent in a reduced ring is central.

Lemma 4.3. Let $A$ be a ring such that $\sigma$ and $D$ commute. Then $\sigma$ and $D$ can be extended to an automorphism of $A[X, \sigma, D]$ and a $\sigma$-derivation on $A[X, \sigma, D]$ defined by $\sigma\left(\sum a_{i} X^{i}\right)=\sum \sigma\left(a_{i}\right) X^{i}$ and $D\left(\sum a_{i} X^{i}\right)=\sum D\left(a_{i}\right) X^{i}$

Proof. By using lemma 3.2, we can show that $\sigma\left(a X^{n} \cdot b X^{m}\right)=\sigma\left(a X^{n}\right) \sigma\left(b X^{m}\right)$ and $D\left(a X^{n} \cdot b X^{m}\right)=D\left(a X^{n}\right) \cdot b X^{m}+\sigma\left(a X^{n}\right) D\left(b X^{m}\right)$ for each $a, b \in A$ and $n, m \in Z \geq 0$. Therefore $\sigma(f g)=\sigma(f) \sigma(g)$ and $D(f g)=D(f) g+\sigma(f) D(g)$ for each $f, g \in A[X, \sigma, D]$. then

Lemma 4.4. Let $A[X, \sigma, D] \cong B[Y, \rho, \delta]$ Via $\Psi$. If $\rho \circ \Psi=\Psi \circ \sigma$ and $\delta \circ \Psi=\Psi \circ D$, 
1) If $\sigma$ and $D$ commute, then $\rho$ and $\delta$ commute.

2) If $A$ is a $\sigma$-reduced ring such that $\sigma$ and $D$ commute, then $B$ is a $\rho$-reduced ring.

Proof. 1) Routine.

2) Let $f(X) \in A[X, \sigma, D]$ be such that $f(X) \sigma(f(X))=0$. Then by using lemma 3.2 we have that

$$
\sum_{k=0}^{2 n}\left[\sum_{i+j=k} a_{i}\left(\sum_{t=0}^{i}\left(\begin{array}{l}
i \\
t
\end{array}\right) \sigma^{i-t} D^{t}\left(\sigma\left(a_{j}\right)\right) X^{i-t}\right) X^{j}\right]=0 .
$$

The coefficient of the highest term in the left-hand expression is $a_{n} \sigma^{n+1}\left(a_{n}\right)$. So since $A$ is $\sigma^{m}$-reduced for each $m=0,1,2, \ldots$ then $a_{n}=0$. Continuing this way we obtain $f(X)=0$ and so $A[X, \sigma, D]$ is a $\sigma$-reduced ring. Therefore we can easily show that $B$ is $\rho$-reduced.

With these lemmas established we are now ready to prove the strong Ore invariance of regular $\sigma$-reduced rings which is the main result of this paper.

Theorem 4.5. Let $A$ be a regular $\sigma$-reduced ring such that $\sigma$ and $D$ commute. If $A[X, \sigma, D] \cong B[Y, \rho, \delta]$ Via $\Psi$ such that $\rho \circ \Psi=\Psi \circ \sigma$ and $\delta \circ \Psi=\Psi \circ D$, then $\Psi(A)=B$.

Proof. By using lemma 4.4, we have $B$ is $\rho$-reduced such that $\rho$ and $\delta$ commute. Therefore proposition 3.4 and lemma 4.2 imply that the set of all units (idempotents) in $A[X, \sigma, D]$ is the set of all units (idempotents) in $A$ and the same is true for $B[Y, \rho, \delta]$ and $B$. By observing that every element of an abelian regular ring is a product of a unit and a central idempotent, we have $\Psi(A) \subseteq B$.

Now let $P$ be a prime ideal of $A$ and set $P^{\prime}=\Psi(P) B$. Since $P$ is generated as an $A$-module by central idempotents, $P^{\prime}$ is also generated by central idempotents. From (2.2), the relations $\rho(\Psi(a))=\Psi(\sigma(a))$ and $\delta(\Psi(a))=\Psi(D(a))$ for each $a \in A$, imply that $P^{\prime}$ is a $\rho$-ideal and $\delta$-ideal of $B$. In this case it is easy to see that $P^{\prime}$ is a proper ideal of $B$. Since $\Psi(P) \subseteq P^{\prime}, \sigma(P)=P, \rho\left(P^{\prime}\right)=P^{\prime}, D(P) \subseteq P$ and $\delta\left(P^{\prime}\right) \subseteq P^{\prime}$, then $\Psi$ induces a ring homomorphism $\bar{\Psi}$ from $(A / P)[X, \bar{\sigma}, \bar{D}] \cong A[X, \sigma, \delta] / P A[X, \sigma, D]$ to $\left(B / P^{\prime}\right)[Y, \bar{\rho}, \bar{\delta}] \cong B[Y, \rho, \delta] / P^{\prime} B[Y, \rho, \delta]$, where $\bar{\sigma}$ and $\bar{\rho}$ are the automorphisms of $A / P$ and $B / P^{\prime}$ respectively induced by $\sigma$ and $\rho$, also $\bar{D}$ and $\bar{\delta}$ are the $\bar{\sigma}$-derivation on $A / P$ and the $\bar{\rho}$-derivation on $B / P^{\prime}$ respectively induced by $D$ and $\delta$. In this case $\bar{\Psi}$ is an onto mapping and $\bar{\Psi}(A / P) \subseteq B / P^{\prime}$.

Since $A$ is abelian regular, then $A / P$ is a division ring and so $(A / P)[X, \bar{\sigma}, \bar{D}]$ is a principal ideal domain by (2.6). Thus if $\operatorname{Ker} \bar{\Psi} \neq 0$, then we have $(A / P)[X, \bar{\sigma}, \bar{D}] / \operatorname{Ker} \bar{\Psi}$ is a finite dimensional vector space over $A / P$. But since $(A / P)[X, \bar{\sigma}, \bar{D}] / \operatorname{Ker} \bar{\Psi} \cong$ $\left(B / P^{\prime}\right)[Y, \bar{\rho}, \bar{\delta}]$ and $\bar{\Psi}(A / P) \subseteq B / P^{\prime}$, this is impossible. Therefore $\operatorname{Ker} \bar{\Psi}=0$ and so $\bar{\Psi}$ is an isomorphism. Since $A / P$ is a division ring, we have $\bar{\Psi}(A / P)=B / P^{\prime}$ by 
proposition 4.1. So $P^{\prime}=\Psi(p) B$ is a maximal ideal of $B$ and $B / P^{\prime}$ is a division ring whenever $P$ is a prime ideal of $A$.

As in the proof of [1, Theorem 3] we can show that every prime factor of the reduced ring $B$ is regular, so $B$ is abelian regular. Since all idempotents and all units of $B$ are in $\Psi(A)$, we have $\Psi(A)=B$.

We conclude this paper by the following remark.

Remark. The structure theorems for finitely generated projective modules over a differential operator ring $A[X, D]$ of abelian regular $\operatorname{ring} A$ obtained in [1] can be extended to finitely generated projective modules over an Ore extension $A[X, \sigma, D]$ in the case where $A$ is a regular $\sigma$-reduced ring. Moreover the proofs are essentially the same as that used in [1].

\section{References}

[1] E. P. Armendariz, H. K. Koo and J. K. Park, "Isomorphic Ore extensions," Communications in Algebra, 15 (12), 2633-2652, 1987.

[2] J. W. Brewer and E. A. Rutter, "Isomorphic polynomial rings," Arch. Math., 23, 484-488, 1972.

[3] D. B. Coleman and E. E. Enochs, "Isomorphic polynomial rings," Proc. Amer. Math. Soc., 27, 24.7-252, 1971.

[4] P. Eakin and K. K. Kubota, "A note on the uniqueness of rings of coefficients in polynomial rings," Proc. Amer. Math. Soc., 32(2), 1972.

[5] M. Ferrero, K. Kishimoto and K. Motose, "On radicals of skew polynomial rings of derivation type," J. of the London Math. Soc., 28(1), 9-16, 1982.

[6] K. R. Goodearl, "Prime ideals in skew polynomial rings and Quantized Weyl Algebras," Journal of Algebra, Vol.150, No. 2, 324-377 August 15, 1992.

[7] J. M. Goursaud, Sur les anneaux introduits par la notion de module Projectif, Théses Présentee a'1'Université de Poitiérs, 1977.

[8] M. Hochster, Nonuniqueness of coefficient rings in a Polynomial ring, Proc. Amer. Math. Soc., 34, 81-82, 1972.

[9] A. A. M. Kamal, "Idempotents in Polynomial rings," Acta Math. Hung., 59(3-4), 355-363, 1992.

[10] A. Leroy and J. Matczuk, "Prime ideals of Ore extensions," Comm. in Alg., (19), 1893-1907, 1991.

[11] J. C. Mcconnell and J. C. Robson, Non commutative Noetherian rings, John Wiley, 1987.

[12] M. Rimmer and K. R. Pearson, "Nilpotents and units in skew polynomial rings over commutative rings," J. Austral. Math. Soc., 28, 423-426, 1979.

Cairo University, Faculty of Science, Department of Mathematics Giza, Egypt. 